\title{
Localization of Sympathetic, Parasympathetic and Sensory Neurons Innervating the Heart of the Beijing Duck by Means of the Retrograde Transport of Horseradish Peroxidase
}

\author{
Yaoxing CHEN, Dacheng LIN, Yasushige OHMORI ${ }^{1)}$ and Jumpei NAITO ${ }^{1)}$ \\ Laboratory of Veterinary Anatomy, College of Veterinary Medicine, China Agricultural University, Beijing 100094, China and \\ ${ }^{1)}$ Laboratory of Animal Morphology and Function, Division of Biofunctions Development, Department of Biological Mechanisms and \\ Functions, Graduate School of Bioagricultural Sciences, Nagoya University, Chikusa-ku, Nagoya 464-8601, Japan
}

(Received 17 June 1998/Accepted 24 August 1998)

ABSTRACT. Sympathetic, parasympathetic and sensory neurons were labeled by injections of horseradish peroxidase into various regions of the heart in 33 Beijing ducks. Sympathetic postganglionic neurons innervating the heart were located in the paravertebral ganglia $\mathrm{C} 15$ (C16 is the last cervical segment in the duck) to T3, especially in the ganglion T1. The coronary sulcus and ventricle were more abundantly innervated by sympathetic neurons than the atrium. The left side of the heart was preferentially innervated by sympathetic postganglionic neurons in the left side of paravertebral ganglia but the right side of the heart were equally supplied from the right and left ganglia. Within the medulla oblongata, the number of labeled vagal preganglionic neurons in the nucleus ambiguus was much greater than that in the dorsal motor nucleus of the vagus nerve. Labeled neurons of the nucleus ambiguus were found in many ducks injected into the coronary sulcus. Cardiac sensory neurons were observed in the dorsal root ganglia C15 to T2 (highest in the ganglion T1) and in the nodose and jugular ganglia of the vagus nerve. These labeled neurons probably form the afferent and efferent limbs of cardiac reflexes and control circulation in the Beijing duck.-KEY wORDS: autonomic nervous system, Beijing duck, heart, parasympathetic neuron, sympathetic neuron.

J. Vet. Med. Sci. 61(1): 1-5, 1999

In the heart of birds, like that of mammals, heart rate is set by the interplay of sympathetic and parasympathetic influences on the pacemaker. Stimulation of the sympathetic cardiac nerve typically increases heart rate, whereas stimulation of the parasympathetic vagus nerve reduces it [17]. There seems to be considerable species variation in the amount of vagal restraint of the heart. Species with a large heart in relation to body size like pigeons, most carnivorous birds, gulls, and ducks have a marked vagal tonus to the heart, while for species like chickens the tonic vagal activity is considerably smaller. This generalization appears to be true at least for the duck and chicken, since bilateral vagotomy of the duck increases heart rate by $65-$ $200 \%$, whereas the same procedure in the chicken increases heart rate by only $20-36 \%$ [17]. These species differences are related to the activity pattern of the birds in normal life.

The distribution of sympathetic, parasympathetic and sensory neurons innervating the avian heart was only studied in the pigeon using retrograde axonal transport of horseradish peroxidase (HRP) [3], electrical stimulation [7, 25] and retrograde degeneration [8, 20]. These studies demonstrated that sympathetic postganglionic neurons were located mainly in the paravertebral ganglion C14 (the last cervical segment in the pigeon), parasympathetic preganglionic neurons were observed in the dorsal motor nucleus of the vagus nerve and sensory neurons were found in the dorsal root ganglia $\mathrm{C} 13$ and $\mathrm{C} 14$. However, using the fragment $\mathrm{C}$ of tetanus toxin as the transport marker molecule, it was demonstrated that the majority of parasympathetic preganglionic neurons innervating the heart of the pigeon were located in the nucleus ambiguus [4].

Recently, distributions of the cardiac plexus and cardiac ganglia were gross-anatomically studied in the Beijing duck [5]. Six cardiac plexuses and cardiac ganglia arising from the sympathetic trunk and the vagus nerve made the particular pattern of the autonomic nerve networks on the heart of the duck. However, few transport studies have been carried out in the duck. The present study aims at elucidating the precise anatomical distribution of sympathetic postganglionic, parasympathetic preganglionic and sensory neurons innervating each cardiac region of the Beijing duck using retrograde axonal transport of HRP.

\section{MATERIALS AND METHODS}

Thirty-three Beijing ducks (Anas platyrhynchos var. domesticus) weighing 1-2 kg (1-2 months old) were used in this study. Under anesthesia with $30 \%$ solution of urethane $(0.8 \mathrm{~g} / \mathrm{kg}$, i.v. $)$, the heart was exposed and $20 \%$ HRP (Sigma, Type VI) was injected into the subepicardium in 1 or 3 regions of the right and left atriums, right and left ventricles and right coronary sulcus (Table 1). Injections of HRP were performed at 5 points ( $5 \mu \mathrm{l} /$ point) in each cardiac region for 10-25 min. After a postinjection survival time of $30-36 \mathrm{hr}$, ducks were deeply anesthetized with $30 \%$ solution of urethane $(1 \mathrm{~g} / \mathrm{kg}$, i.v.) and perfused with $0.75 \%$ saline $\left(40^{\circ} \mathrm{C}\right)$ through the left ventricle, followed by the fixative consisting of $2 \%$ paraformaldehyde and $1.25 \%$ 
Table 1. The number of labeled neurons in the paravertebral, dorsal root, nodose and jugular ganglia and in the medulla oblongata after injections of HRP into the heart of the Beijing duck

\begin{tabular}{|c|c|c|c|c|c|c|c|c|c|c|c|c|c|c|c|c|c|c|c|c|c|c|c|c|c|c|c|}
\hline \multirow{2}{*}{$\begin{array}{l}\text { Duck } \\
\text { No. }\end{array}$} & \multirow{2}{*}{$\begin{array}{l}\text { Injection } \\
\text { site }\end{array}$} & \multicolumn{5}{|c|}{ Right paravertebral ganglia } & \multicolumn{5}{|c|}{ Left paravertebral ganglia } & \multirow{2}{*}{$\begin{array}{l}\text { Right } \\
\text { DMNV }\end{array}$} & \multirow{2}{*}{$\begin{array}{c}\text { Left } \\
\text { DMNV }\end{array}$} & \multirow{2}{*}{$\begin{array}{l}\text { Right } \\
\text { NA }\end{array}$} & \multirow{2}{*}{$\begin{array}{l}\text { Left } \\
\text { NA }\end{array}$} & \multicolumn{4}{|c|}{ Right dorsal root ganglia } & \multicolumn{4}{|c|}{ Left dorsal root ganglia } & \multirow{2}{*}{$\begin{array}{l}\text { Right } \\
2 \mathrm{NG}\end{array}$} & \multirow{2}{*}{$\begin{array}{l}\text { Left } \\
\text { NG }\end{array}$} & \multirow{2}{*}{$\begin{array}{c}\text { Right } \\
\text { JG }\end{array}$} & \\
\hline & & $\mathrm{C} 15$ & C16 & $\mathrm{T} 1$ & $\mathrm{~T} 2$ & $\mathrm{~T} 3$ & $\mathrm{C} 15$ & C16 & $\mathrm{T} 1$ & $\mathrm{~T} 2$ & $\mathrm{~T} 3$ & & & & & C15 & $\mathrm{C} 16$ & $\mathrm{~T} 1$ & $\mathrm{~T} 2$ & C15 & $\mathrm{C} 16$ & $\mathrm{~T} 1$ & $\mathrm{~T} 2$ & & & & \\
\hline D13 & RA & - & - & 3 & - & - & - & - & 4 & - & - & - & - & - & - & - & - & - & - & - & - & - & - & - & - & - & - \\
\hline D50 & RA & - & - & 8 & - & - & - & - & 3 & - & - & - & - & - & - & - & - & - & - & - & - & - & - & - & - & - & - \\
\hline D51 & RA & - & - & 2 & - & - & - & - & 2 & - & - & - & - & - & - & - & - & - & - & - & - & - & - & - & - & - & - \\
\hline D61 & RA & - & - & 3 & 2 & - & - & - & - & 3 & - & - & - & 2 & - & - & - & - & - & - & - & - & - & - & - & - & - \\
\hline D34 & RCS & - & 34 & 336 & 177 & - & - & 48 & 194 & 15 & 1 & - & - & 3 & 3 & - & 2 & 29 & 2 & - & 4 & 4 & 4 & - & 1 & - & - \\
\hline D35 & RCS & - & - & - & - & - & - & - & - & - & - & - & 1 & 4 & 3 & - & - & - & - & - & - & - & - & - & - & - & - \\
\hline D36 & RCS & - & - & - & - & - & - & - & - & - & - & - & - & - & - & - & - & - & - & - & - & - & - & - & 1 & - & - \\
\hline D41 & RCS & - & - & 37 & 29 & - & - & - & 39 & 18 & - & - & - & - & - & - & - & - & 1 & - & - & - & - & 2 & 2 & - & - \\
\hline D63 & RCS & - & 3 & 20 & 2 & - & - & 13 & 37 & 10 & - & 6 & - & 2 & 1 & - & - & - & - & - & - & - & - & - & 19 & - & - \\
\hline D64 & RCS & - & 25 & 61 & 1 & - & - & 12 & 14 & 2 & - & 11 & 15 & 9 & 8 & - & 1 & 1 & 1 & - & 3 & 7 & - & - & - & - & - \\
\hline D20 & $\mathrm{RA}+\mathrm{RCS}$ & - & - & 4 & 1 & - & - & 8 & 34 & 6 & - & 1 & 2 & 6 & 6 & - & - & - & - & - & - & - & - & - & 6 & - & 11 \\
\hline D22 & $\mathrm{RA}+\mathrm{RCS}$ & - & - & - & - & - & - & - & - & - & - & - & 2 & 1 & 2 & - & - & - & - & - & - & - & - & - & - & - & - \\
\hline D23 & $\mathrm{RA}+\mathrm{RCS}$ & - & - & - & - & - & - & - & - & - & - & - & 1 & - & 1 & - & - & - & - & - & 4 & 20 & - & - & - & - & - \\
\hline D25 & $\mathrm{RA}+\mathrm{RCS}$ & - & 11 & 32 & 3 & - & - & 2 & 36 & 4 & - & - & - & 5 & 2 & - & - & - & 1 & - & - & - & - & 2 & 4 & - & - \\
\hline D26 & $\mathrm{RA}+\mathrm{RCS}$ & - & - & - & - & - & - & - & - & - & - & - & - & - & - & - & - & 2 & - & - & - & - & - & 2 & 1 & - & - \\
\hline D27 & $\mathrm{RA}+\mathrm{RCS}$ & - & - & - & - & - & - & - & - & - & - & - & - & 2 & 4 & - & - & - & - & - & - & - & - & - & - & - & 7 \\
\hline D29 & $\mathrm{RA}+\mathrm{RCS}$ & - & - & - & - & - & - & - & - & - & - & - & 1 & 3 & 4 & - & - & - & - & - & - & - & - & - & 1 & - & - \\
\hline D39 & $\mathrm{RA}+\mathrm{RCS}$ & - & 13 & 13 & 30 & - & - & 6 & 4 & 10 & - & - & - & - & - & - & - & - & 1 & - & - & - & 1 & 3 & - & - & - \\
\hline D40 & $\mathrm{RA}+\mathrm{RCS}$ & - & 6 & 28 & 1 & - & - & 1 & 175 & - & - & - & - & - & - & - & - & 3 & - & - & - & 1 & - & 2 & - & - & - \\
\hline D4 & RV & - & 1 & 27 & 6 & - & - & 6 & 12 & - & - & - & - & - & - & - & - & - & - & - & - & - & 4 & - & 8 & - & - \\
\hline D8 & RV & - & 10 & 18 & 4 & - & - & 4 & 12 & 3 & - & - & - & - & - & - & - & - & - & - & - & - & - & - & 3 & - & - \\
\hline D9 & RV & - & 7 & 62 & - & - & - & 15 & 137 & 9 & - & - & - & - & - & - & - & - & - & - & - & - & - & - & 5 & - & - \\
\hline D52 & RV & - & 33 & 118 & 23 & - & - & 22 & 123 & 59 & - & - & - & - & - & - & 16 & 9 & 3 & - & 6 & 23 & 7 & - & 3 & - & - \\
\hline D42 & All R & - & 3 & 83 & 8 & - & - & 11 & 90 & 20 & - & 7 & 7 & 15 & 16 & - & - & - & 3 & - & - & - & 4 & 2 & 8 & - & - \\
\hline D31 & LA & - & - & - & - & - & - & - & 13 & 5 & - & - & - & - & - & - & - & - & - & - & - & - & - & 4 & - & - & - \\
\hline D46 & LA & - & - & 3 & - & - & - & - & 6 & - & - & - & - & - & - & - & - & - & - & - & - & - & - & - & - & - & - \\
\hline D55 & LA & - & 6 & 10 & - & - & 15 & 4 & 15 & - & - & - & - & - & - & - & - & 1 & - & 2 & - & 5 & - & - & - & - & - \\
\hline D57 & LA & - & - & - & - & - & - & 1 & 5 & - & - & - & - & - & - & - & - & - & - & - & - & - & - & - & - & - & - \\
\hline D33 & LV & - & 1 & 13 & 3 & - & - & 4 & 24 & 171 & - & - & - & - & - & - & - & - & 1 & - & - & - & - & - & - & - & - \\
\hline D44 & LV & - & 84 & 77 & 3 & - & - & 100 & 331 & 171 & - & - & - & - & - & - & - & - & - & - & 8 & 17 & 24 & - & 2 & - & - \\
\hline D45 & LV & - & - & 8 & - & - & - & 1 & 13 & - & - & - & - & - & - & - & - & - & - & - & - & - & - & - & - & - & 1 \\
\hline D47 & LV & - & - & 31 & 7 & - & - & 6 & 105 & 45 & 2 & 2 & - & 4 & 3 & - & - & - & - & - & - & - & - & 2 & - & - & - \\
\hline D48 & LV & - & - & - & - & - & - & - & - & - & - & - & - & 10 & 5 & - & - & - & - & - & - & - & 2 & - & - & - & - \\
\hline
\end{tabular}

All R: RA+RCS+RV; DMNV: dorsal motor nucleus of the vagus nerve; JG: jugular ganglion; LA: left atrium; LV: left ventricle; NA: nucleus ambiguus; NG: nodose ganglion; RA: right atrium; RCS: right coronary sulcus; RV: right ventricle; -: no labeled neurons.

glutaraldehyde in $0.1 \mathrm{M}$ phosphate buffer ( $\mathrm{pH}$ 7.4). Finally, ducks were perfused with $5 \%$ sucrose in $0.1 \mathrm{M}$ phosphate buffer at $4{ }^{\circ} \mathrm{C}$. After perfusion, the spinal segments $\mathrm{C} 2$ to T6 together with the dorsal root ganglia and paravertebral ganglia, the nodose and jugular ganglia and the brainstem were immediately removed, and these tissues were immersed overnight in phosphate-buffered $30 \%$ sucrose at $4^{\circ} \mathrm{C}$. Serial frozen sections were cut at $40 \mu \mathrm{m}$ in thickness with a cryostat microtome. The sections were processed for HRP histochemistry with benzidine dihydrochloride as chromogen [22] and mounted on glass slides in $2 \%$ gelatin and $50 \%$ ethanol solution. After drying in air, selected sections were lightly counterstained with $1 \%$ neutral red solution.

\section{RESULTS}

After injections of HRP into the heart of the duck, retrogradely labeled neurons were bilaterally found in paravertebral ganglia, in the dorsal motor nucleus of the vagus nerve and the nucleus ambiguus of the medulla oblongata, in dorsal root ganglia, and in the nodose and jugular ganglia of the vagus nerve (Table 1). Labeled neurons were not found in any regions of the spinal cord. These findings are described in detail below.

Sympathetic postganglionic neurons: After injections of HRP into the various regions of the heart in the duck, labeled neurons were mainly found in the paravertebral ganglia C16 (the last cervical segment in the duck) to T2 of the 
sympathetic trunk. The number of labeled neurons was found to be highest in the paravertebral ganglion T1 (about two-thirds of the labeled neurons), followed by the ganglia $\mathrm{T} 2$ and $\mathrm{C} 16$ in that order. Only in three experiments, labeled neurons were also observed in the paravertebral ganglion $\mathrm{C} 15$ or T3. Following injections into the coronary sulcus or ventricle, the number of labeled neurons was greater than that after injections into the atrium. After injections of HRP into the right side of the heart, some ducks contained more labeled neurons in the right paravertebral ganglia than in the left ganglia and others had more labeled neurons in the left ganglia than in the right ganglia. On the other hand, the ducks injected into the left side of the heart always contained a greater number of labeled neurons in the left paravertebral ganglia than in the right ganglia. Labeled neurons in the paravertebral ganglia were mainly polygonal in shape (Fig. 1). The somal size of labeled neurons was variable but the large majority were small and mediumsized neurons (15-25 $\mu \mathrm{m}$ in diameter).

Vagal preganglionic neurons: In 14 of the 33 ducks, labeled neurons were found in the medulla oblongata of many experiments, especially after injections of HRP into the coronal sulcus. About two-thirds of labeled neurons observed within the medulla oblongata were located in the nucleus ambiguus and the rest were found in the ventrolateral part of the dorsal motor nucleus. Labeled neurons were observed on the right and left sides of both nuclei between $2.0 \mathrm{~mm}$ rostral to the obex and $0.4 \mathrm{~mm}$ caudal to the obex, respectively. Labeled neurons in the dorsal motor nucleus of the vagus nerve were spindle in shape (Fig. 2) but labeled neurons in the nucleus ambiguus were polygonal in shape (Fig. 3). The size of the labeled neurons in the nucleus ambiguus (20-30 $\mu \mathrm{m}$ in diameter) was larger than that of the labeled neurons in the dorsal motor nucleus (18-20 $\mu \mathrm{m}$ in diameter).

Sensory neurons: In 14 of the 33 ducks, labeled neurons were found in the dorsal root ganglia $\mathrm{C} 16$ to $\mathrm{T} 2$ on both sides after injections of HRP into the various regions of the heart. The number of labeled neurons was found to be highest in the dorsal root ganglion $\mathrm{T} 1$ (about a half of the labeled neurons), followed by the ganglia $\mathrm{T} 2$ and $\mathrm{C} 16$ in that order. In only one duck, labeled neurons were also observed in the dorsal root ganglion C15. These labeled neurons were preferentially located at the central part of each ganglion. After injections into the right side of the heart, labeled neurons were found in the right and left dorsal root ganglia. On the other hand, labeled neurons were exclusively detected in the ipsilateral ganglia after injections into the left side of the heart. Labeled neurons in the dorsal root ganglia were round or oval in shape (Fig. 4). More than $70 \%$ of labeled neurons
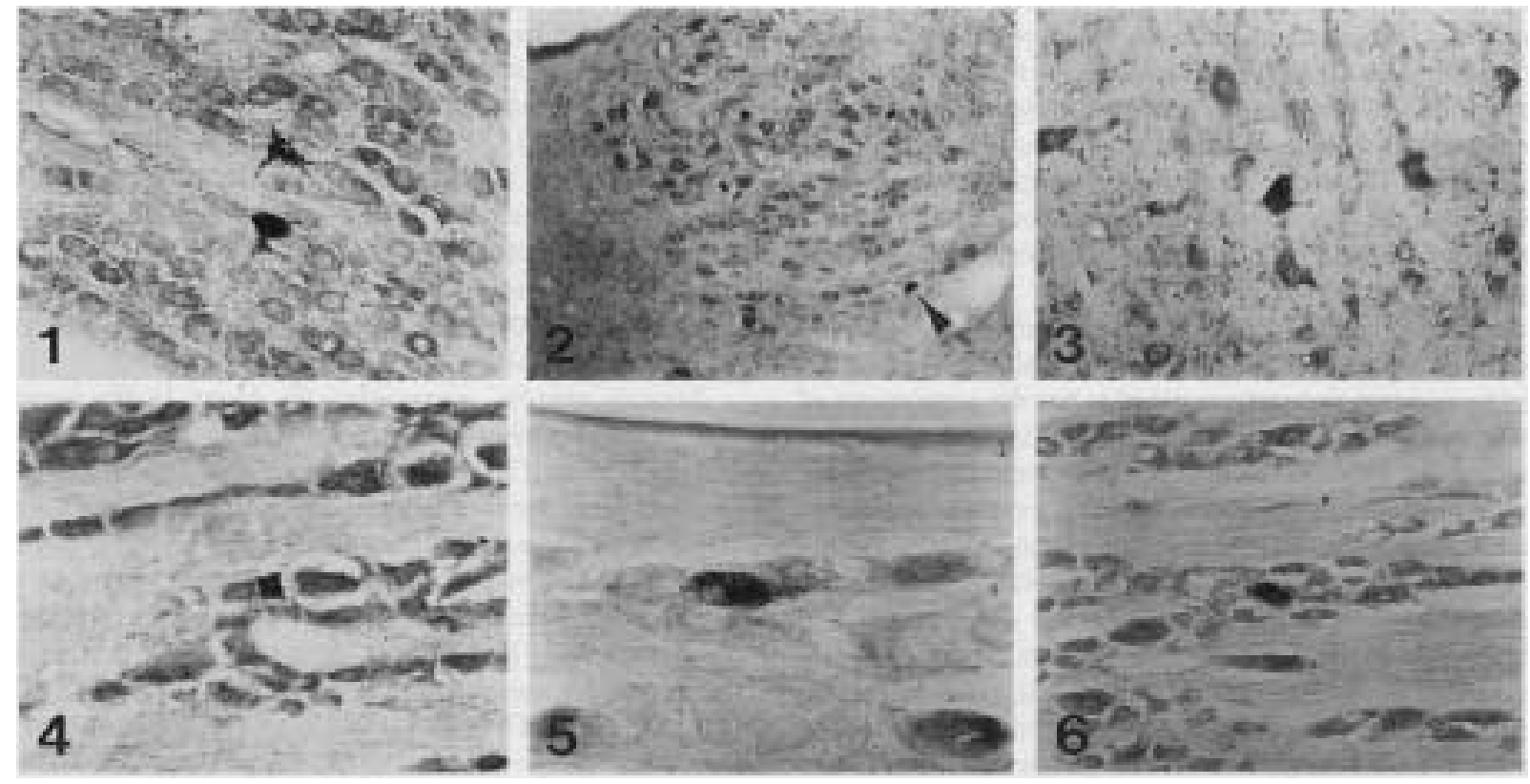

Fig. 1. Labeled sympathetic postganglionic neurons in the right paravertebral ganglion T1 after injection of HRP into the right coronary sulcus of the heart. $\times 125$.

Fig. 2. Labeled parasympathetic preganglionic neurons (arrowhead) in the right dorsal motor nucleus of the vagus nerve after injection of HRP into the right coronary sulcus of the heart. $\times 62.5$.

Fig. 3. Labeled vagal preganglionic neurons in the right nucleus ambiguus after injection of HRP into the right coronary sulcus of the heart. $\times 125$.

Fig. 4. Labeled sensory neurons in the left dorsal root ganglion C16 after injection of HRP into the left ventricle of the heart. $\times 125$.

Fig. 5. Labeled sensory neurons in the right nodose ganglion after injection of HRP into the left ventricle of the heart. $\times 125$.

Fig. 6. Labeled sensory neurons in the left jugular ganglion after injection of HRP into the right atrium and coronary sulcus of the heart. $\times 125$. 
were small cells (10-20 $\mu \mathrm{m}$ in diameter).

In 20 of the ducks, labeled neurons were also found in the vagal nodose and/or jugular ganglia. Labeled neurons were observed in the nodose ganglion of many experiments, especially after injections into the right coronal sulcus and/ or ventricle. Most of the labeled neurons in the nodose ganglion were ellipsoid in shape ( $40 \mu \mathrm{m}$ in length and 20 $\mu \mathrm{m}$ in width) and located in the peripheral zone of the ganglion (Fig. 5). On the other hand, labeled neurons in the jugular ganglion were detected only in the 3 experiments. They were exclusively located in the left ganglion. Labeled neurons were middle-sized cells $(20-25 \mu \mathrm{m}$ in diameter) and round in shape (Fig. 6).

\section{DISCUSSION}

Sympathetic postganglionic neurons innervating the heart: In our previous gross-anatomical study, the cardiac sympathetic nerve of the duck was observed to arise from the parasympathetic ganglion $\mathrm{T} 1$ or $\mathrm{T} 2$, but not from the cervical parasympathetic ganglia [5]. In the present study, however, sympathetic postganglionic neurons innervating the heart were observed in the paravertebral ganglia C15 to $\mathrm{T} 3$, especially in the ganglion T1. After transection of the sympathetic cardiac nerve in the pigeon, chromatolytic neurons were observed in the paravertebral ganglia $\mathrm{C} 12$ to $\mathrm{C} 14$ and the proportion of such cells was highest in the ganglion $\mathrm{C} 14$, intermediate in the ganglion $\mathrm{C} 13$ and lowest in the ganglion C12 [20]. Moreover, HRP-labeled neurons were found in the paravertebral ganglion $\mathrm{C} 14$ following application of HRP to the sympathetic cardiac nerve of the pigeon [3]. Therefore, sympathetic postganglionic neurons innervating the heart of birds are situated in the paravertebral ganglia of the most caudal cervical and most cranial thoracic segments.

In mammals, the location of sympathetic postganglionic neurons innervating the heart are mainly present in the middle cervical and stellate ganglia, but the proportions of postganglionic neurons in these ganglia differ between mammalian species. In the dog, they were located in the greatest number in the middle cervical ganglion and fewer were located in the stellate ganglion $[1,10,12,30]$. On the other hand, postganglionic neurons innervating the cat heart were predominantly found in the stellate ganglion $[18,19$, $23,26,32]$. In the pig, these neurons were relatively evenly distributed among the stellate and middle cervical ganglia [15]. Although paravertebral ganglia in birds do not fuse to form the middle cervical or stellate ganglion, the difference in craniocaudal distribution of postganglionic neurons innervating the heart was also found between avian species. Postganglionic neurons innervating the duck heart seem to be located in more caudal segments of paravertebral ganglia than those innervating the pigeon heart.

In the present study, the right side of the heart was bilaterally innervated by postganglionic neurons of paravertebral ganglia but the left side of the heart was ipsilateral-dominantly innervated. There was a difference in sympathetic innervation between the right and left sides of the heart. This may be related to functional differences between the right and left sympathetic cardiac nerves. In the pigeon, electrical stimulation of the right cardiac nerve consistently increases heart rate, whereas this is not true for the left [20]. In parasympathetic and sensory innervation to the heart of the duck, we could not detect obvious laterality.

Parasympathetic preganglionic neurons innervating the heart: Previous studies using electrical mapping methods [7], retrograde degeneration [8] or single unit studies [25] demonstrated that vagal preganglionic neurons innervating the heart of the pigeon were located in the dorsal motor nucleus of the vagus nerve. No evidence of chromatolysis was found in the nucleus ambiguus [8]. Thus, parasympathetic preganglionic neurons innervating the heart of birds were thought to be within the dorsal motor nucleus of the vagus nerve. On the other hand, in a retrograde labeling study using fragment $\mathrm{C}$ of tetanus toxin, it was demonstrated that the majority of labeled vagal preganglionic neurons innervating the heart of the pigeon were confined to the nucleus ambiguus and a smaller number of labeled neurons were located in the dorsal motor nucleus of the vagus nerve [4]. In the present study, a quantitative majority of neurons innervating the heart of the duck were located in the nucleus ambiguus and not in the dorsal motor nucleus as previously thought. In mammals, vagal preganglionic neurons innervating parasympathetic postganglionic neurons of the heart have been found in 2 separate regions of the medulla oblongata; the dorsal motor nucleus of the vagus nerve and the nucleus ambiguus [cat: 2, 6, 9, 18, 21, 23, 29; dog: 2, 11; monkey: 14; pig: 15, 16; rat: $24,27,28]$. A quantitative majority of preganglionic neurons are located in the nucleus ambiguus and ventrolateral nucleus ambiguus. Therefore, it is suggested that the cardiac parasympathetic preganglionic representation in the avian medulla oblongata is very similar to that in mammals.

Sensory neurons innervating the heart: In the present study, sensory neurons were labeled in the dorsal root ganglia $\mathrm{C} 15$ to $\mathrm{T} 2$ and in the nodose and jugular ganglia. In the pigeon, sensory neurons innervating the heart were found in the dorsal root ganglia C13 and C14 [3], but the presence of nodose and jugular ganglion cells supplying the heart has not been reported in any other species of birds as far as we know. In mammals, sensory neurons innervating the heart were observed in the dorsal root ganglia C8 toT9 [cat: 19], C8 to T6 [cat: 31] or C6 to T6 [dog: 13], and in the nodose ganglion [cat: 18, 23; dog: 13; pig: 15]. These sensory neurons form the afferent limbs of cardiac reflexes, transmitting sensory impulses from the heart associated with the control of circulation.

ACKNOWLEDGMENTS. We thank Prof. K. Fukuta for a sincere encouragement in achieving this study. This study was supported in part by Grant-in-Aid for Scientific Research (No. 07306025, Research-leader: Prof. S. Sugano) from the Ministry of Education, Science and Culture, Japan. 


\section{REFERENCES}

1. Armour, J. A. and Hopkins, D. A. 1981. Localization of sympathetic postganglionic neurons of physiologically identified cardiac nerves in the dog. J. Comp. Neurol. 202: 169-184.

2. Bennett, J. A., Kidd, C., Latif, A. B. and McWilliam, P. N. 1981. A horseradish peroxidase study of vagal motoneurones with axons in cardiac and pulmonary branches of the cat and dog. Q. J. Exp. Physiol. 66: 145-154.

3. Cabot, J. B. and Cohen, D. H. 1977. Anatomical and physiological characterization of avian sympathetic cardiac afferents. Brain Res. 131: 89-101.

4. Cabot, J. B., Carroll, J. and Bogan, N. 1991. Localization of cardiac parasympathetic preganglionic neurons in the medulla oblongata of pigeon, Columba livia: a study using fragment C of tetanus toxin. Brain Res. 544: 162-168.

5. Chen, Y., Lin, D., Ohmori, Y. and Naito, J. 1997. Distributions of the cardiac plexuses and ganglia in the Beijing duck. J. Vet. Med. Sci. 59: 409-411.

6. Ciriello, J. and Calaresu, F. R. 1982. Medullary origin of vagal preganglionic axons to the heart of the cat. J. Auton. Nerv. Syst. 5: 9-22.

7. Cohen, D. H. and Schnall, A. M. 1970. Medullary cells of origin of vagal cardioinhibitory fibers in the pigeon. II. Electrical stimulation of the dorsal motor nucleus. J. Comp. Neurol. 140: 321-342.

8. Cohen, D. H., Schnall, A. M., Macdonald, R. L. and Pitts, L. H. 1970. Medullary cells of origin of vagal cardioinhibitory fibers in the pigeon. I. Anatomical studies of peripheral vagus nerve and the dorsal motor nucleus. J. Comp. Neurol. 140: 299-320.

9. Geis, G. S. and Wurster, R. D. 1980. Horseradish peroxidase localization of cardiac vagal preganglionic somata. Brain Res. 182: $19-30$.

10. Hirakawa, N., Morimoto, M. and Totoki, T. 1993. Sympathetic innervation of the young canine heart using antero- and retrograde axonal tracer methods. Brain Res. Bull. 31: 673680.

11. Hopkins, D. A. and Armour, J. A. 1982. Medullary cells of origin of physiologically identified cardiac nerves in the dog. Brain Res. Bull. 8: 359-365.

12. Hopkins, D. A. and Armour, J. A. 1984. Localization of sympathetic postganglionic and parasympathetic preganglionic neurons which innervate different regions of the $\operatorname{dog}$ heart. $J$. Comp. Neurol. 229: 186-198.

13. Hopkins, D. A. and Armour, J. A. 1989. Ganglionic distribution of afferent neurons innervating the canine heart and cardiopulmonary nerves. J. Auton. Nerv. Syst. 26: 213-222.

14. Hopkins, D. A. and Armour, J. A. 1998. Brainstem cells of origin of physiologically identified cardiopulmonary nerves in the rhesus monkey (Macaca mulatta). J. Auton. Nerv. Syst. 68: 21-32.

15. Hopkins, D. A., Gootman, P. M., Gootman, N. and Armour, J. A. 1997. Anatomy of medullary and peripheral autonomic neurons innervating the neonatal porcine heart. J. Auton. Nerv. Syst. 64: 74-84.

16. Hopkins, D. A., Gootman, P. M., Gootman, N., DiRusso, S. M. and Zeballos, M. E. 1984. Brainstem cells of origin of the cervical vagus and cardiopulmonary nerves in the neonatal pig (Sus scrofa). Brain Res. 306: 63-72.

17. Jones, D. R. and Johansen, K. 1972. The blood vascular system of birds. pp. 157-285. In: Avian Biology, vol. II (Farner, D. S., King, J. R. and Parkes, K. C. eds.), Academic Press, New York.

18. Kalia, M. and Mesulam, M.-M. 1980. Brain stem projections of sensory and motor components of the vagus complex in the cat: II. Laryngeal, tracheobronchial, pulmonary, cardiac, and gastrointestinal branches. J. Comp. Neurol. 193: 467508.

19. Kuo, D. C., Oravitz, J. J. and de Groat, W. C. 1984. Tracing of afferent and efferent pathways in the left inferior cardiac nerve of the cat using retrograde and transganglionic transport of horseradish peroxidase. Brain Res. 321: 111-118.

20. Macdonald, R. L. and Cohen, D. H. 1970. Cells of origin of sympathetic pre- and postganglionic cardioacceleratory fibers in the pigeon. J. Comp. Neurol. 140: 343-358.

21. Massari, V. J., Johnson, T. A. and Gatti., P. J. 1995. Cardiotopic organization of the nucleus ambiguus? An anatomical and physiological analysis of neurons regulating atrioventricular conduction. Brain Res. 679: 227-240.

22. Mesulam, M.-M. 1976. The blue reaction product in horseradish peroxidase neurohistochemistry: incubation parameters and visibility. J. Histochem. Cytochem. 24: 1273-1280.

23. Miura, M. and Okada, J. 1981. Cardiac and non-cardiac preganglionic neurones of the thoracic vagus nerve: an HRP study in the cat. Jpn. J. Physiol. 31: 53-66.

24. Nosaka, S., Yamamoto, T. and Yasunaga, K. 1979. Localization of vagal cardioinhibitory preganglionic neurons within rat brain stem. J. Comp. Neurol. 186: 79-92.

25. Schwaber, J. S. and Cohen, D. H. 1978. Field potential and single unit analyses of the avian dorsal motor nucleus of the vagus and criteria for identifying vagal cardiac cells of origin. Brain Res. 147: 79-90.

26. Shih, C.-J., Chuang, K.-S., Tsai, S.-H. and Liu, J.-C. 1985. Horseradish peroxidase localization of the sympathetic postganglionic neurons innervating the cat heart. J. Auton. Nerv. Syst. 13: 179-189.

27. Standish, A., Enquist, L. W., Escardo, J. A. and Schwaber, J. S. 1995. Central neuronal circuit innervating the rat heart defined by transneuronal transport of pseudorabies virus. $J$. Neurosci. 15: 1998-2012.

28. Stuesse, S. L. 1982. Origins of cardiac vagal preganglionic fibers: a retrograde transport study. Brain Res. 236: 15-25.

29. Sugimoto, T., Itoh, K., Mizuno, N., Nomura, S. and Konishi, A. 1979. The site of origin of cardiac preganglionic fibers of the vagus nerve: an HRP study in the cat. Neurosci. Lett. 12: $53-58$.

30. Tomney, P. A., Hopkins, D. A. and Armour, J. A. 1985. Axonal branching of canine sympathetic postganglionic cardiopulmonary neurons. A retrograde fluorescent labeling study. Brain Res. Bull. 14: 443-452.

31. Vance, W. H. and Bowker, R. C. 1983. Spinal origins of cardiac afferents from the region of the left anterior descending artery. Brain Res. 258: 96-100.

32. Wu, J.-J., Chuang, K.-S., Shih, C.-J. and Liu, J.-C. 1988. Sympathetic postganglionic innervation of the cardiac coronary artery in cats. J. Auton. Nerv. Syst. 24: 215-220. 